\title{
Research Methods And Experiments of Piezoelectric Plates
}

\author{
Andrzej Wróbel ${ }^{1, *}$ \\ ${ }^{1}$ Department of Engineering Processes Automation and Integrated Manufacturing Systems, Faculty of \\ Mechanical Engineering, Silesian University of Technology, Konarskiego 18a Street, 44-100 Gliwice, \\ Poland
}

\begin{abstract}
The correct design of technical systems requires consideration of both geometrical and material parameters not only of the mechanical part, but also of the electrical part of the system. The correct assessment of characteristics and testing of intelligent systems should be carried out by both theoretical analyzes and laboratory tests. Usually this description is by means of mathematical equations. The ideal solution is in which it is possible to refine the object in such a way that the obtained model is the most accurate, and even the same as the physical model. Considering the complexity of the model, computational cost, computational possibilities and time-consuming calculations would practically prevent the use of this model. Intelligent materials are more often used for active vibration damping. They can be defined as a group of materials that have the ability to change their physical properties in an appropriate way as a result of external influences. One of the most popular materials in this group are piezoelectric materials. They are used where it is necessary to reduce vibrations with small amplitudes of $\mu \mathrm{m}$ and work in high frequency ranges. In the presented article, the author will attempt to refine the model of intelligent materials with particular emphasis on piezoelectric plates.
\end{abstract}

\section{Introduction}

The article attempts to present processing of analysis results of mathematical analyzes with modeling and testing of phenomena occurring in intelligent materials, in particular piezoelectric plates. The submitted studies are part of the conducted research at the Department of Automation of Engineering Processes and Integrated Manufacturing Systems of the Silesian University of Technology. Intelligent materials can be defined as a group of materials that are available to change properties under the influence of external factors. The most popular materials in this group include metal alloys with shape memory, magnetorheological fluids as well as piezoelectric materials. Due to the great possibilities of applications, these materials are often used in various fields of science and technology for several decades. Piezoelectrics are most often used in automatics, micromanipulation, measuring technology and medicine, used as actuators or sensors. One of the disadvantages

\footnotetext{
* Corresponding author: andrzej.wrobel@polsl.pl
} 
of this kind of sensors is the small displacement, which can be increased by complex systems.

The correct identification and analysis of the piezoelectric systems should be carried out both by theoretical analysis and laboratory tests. Under certain conditions, physical examination of vibrating objects is difficult or impossible to implement. In cases where laboratory tests are expensive and their implementation is economically unjustified, a correspondingly similar mathematical model should be designed.

The description of the analyzed physical phenomenon is usually presented by means of mathematical equations. These are most often differential equations, which are a mathematical model of the studied phenomenon. This is usually a continuous or discrete model.

The ideal solution is the situation in which the designer will such elaborate a detailed object, so that the obtained model was the most accurate and even perfect with the physical model. However, considering the complexity of the model, computing capabilities, and time-consuming calculations would make it practically impossible to use this model. In the presented study, the author attempts to rather detail analyze the longitudinally vibrating piezoelectric plate and to examine the influence of external and internal phenomena occurring in it.

This article is a part of undertaken analysis and experimental research of piezoelectric plates by author on physical phenomena occurring in intelligent materials, working as subassemblies or assemblies in machine construction.

\section{Model under examination}

The basic model adopted for analysis by the author is the model of a single piezoelectric plate, as in Figure 1.

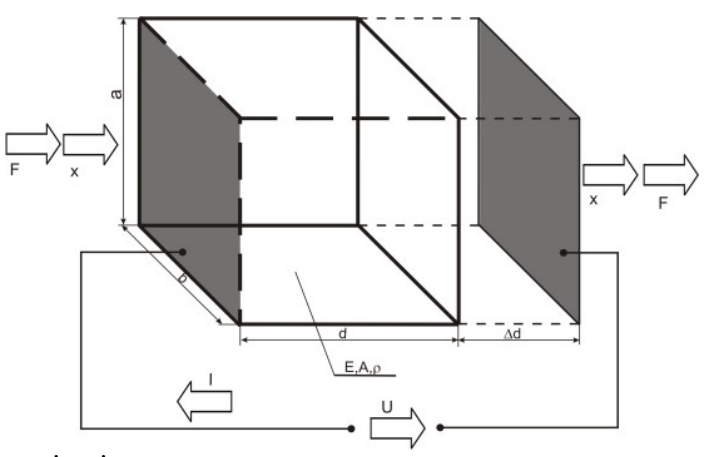

Fig. 1. Model under examination

The accepted model is characterised by the segment constant section. Parameters describing the piezoelement model are: face area, Young's modulus, mass density, power voltage applied to connectors and current. Dislocations (x), and current (I) are caused by exciting forces $(\mathrm{F})$. The exaggerated model is very convenient for mathematical analysis, especially for matrix analysis, in which case the system can be treated as the $\mathrm{n}$ port system. Linear dislocations are measured only on the $\mathrm{x}$ axis starting from the beginning of the co-ordinate system.

Assuming the right matrix [1, 3, 5] containing both mechanical and electrical elements presented in equation 1, it is difficult to apply the already known and previously used methods for the analysis of mechanical systems. 


$$
\left[\begin{array}{c}
F_{1} \\
U \\
F_{2}
\end{array}\right]=\left[\begin{array}{ccc}
\frac{Z}{\tan k d} & \frac{h}{\omega} & -\frac{Z}{\sin k d} \\
\frac{h}{\omega} & \frac{1}{\omega C_{0}} & -\frac{h}{\omega} \\
\frac{Z}{\sin k d} & \frac{h}{\omega} & -\frac{Z}{\tan k d}
\end{array}\right]\left[\begin{array}{c}
u_{1} \\
i \\
u_{2}
\end{array}\right] .
$$

The use of a matrix presented in equation 1 in a detailed analysis of the piezoelectrics may prove to be a time-consuming activity. Therefore, the authors idea was performed modeling of the system using a replacement system. In order to maintain the transparency of a representation, the characteristics are marked, in brackets, by Arabic numbers according to $[2,6,7]$. As a result of introduction of the substitute matrix, there was obtained matrix $2 \times 2$, which is the basis for further analysis by network methods.

In order to apply the method of structural numbers, the piezoelectric plate system should be analyzed in the simplest possible form. The main benefit of such a conversion is the reduction of the four-vertex graph (Fig 2.) being a representation of the first system, minimization of the number of branches and vertexes, with constant input-output relations.

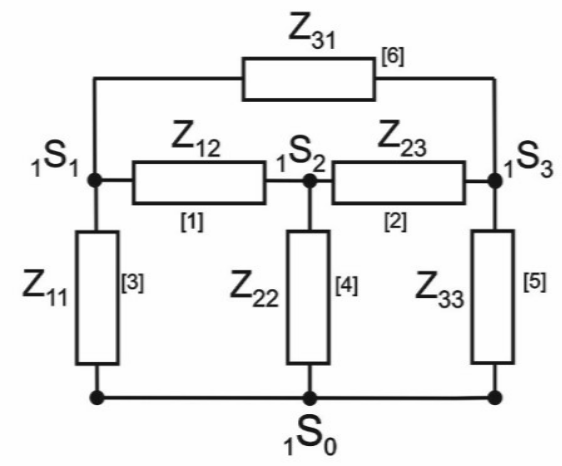

Fig. 2. The representation of the first system for analysis

As a result of the reduction system (Fig. 2) into the reduced system we expect to receive a system shown in Fig. 3.

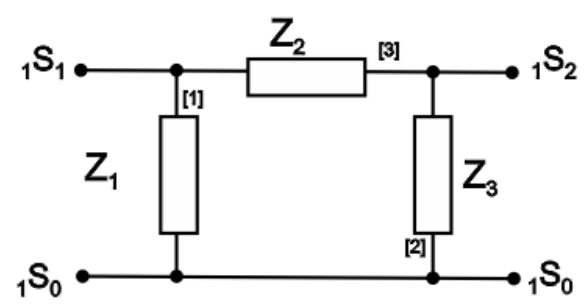

Fig. 3. The representation of the replacement circuit accepted for further analysis

The structural number of the oryginal system is: 


$$
A=[136][256][345] \text {. }
$$

By multiplying the factors of the structural number (2), we obtained:

$$
A=\left[\begin{array}{llllllllllllllllll}
1 & 1 & 1 & 1 & 1 & 1 & 1 & 1 & 3 & 3 & 3 & 3 & 3 & 6 & 6 & 6 & 6 & 6 \\
2 & 2 & 2 & 5 & 5 & 6 & 6 & 6 & 2 & 2 & 5 & 6 & 6 & 2 & 2 & 2 & 5 & 5 \\
3 & 4 & 5 & 3 & 4 & 3 & 4 & 5 & 4 & 5 & 4 & 4 & 5 & 3 & 4 & 5 & 3 & 4
\end{array}\right]
$$

The edges of the replacement graph were calculated based on the formula:

$$
Z_{k}=\frac{\operatorname{det}\left(\frac{\partial A}{\partial D^{\mu_{1} k}} \cap \frac{\partial A}{\partial D^{\mu_{2} k}}\right)}{\operatorname{det}_{z} \frac{\partial A}{\partial D}} .
$$

Using the signs shown in Equation 4, there was written:

$$
Z_{1^{\prime}}=\frac{\operatorname{det}\left(A_{2^{\prime}} \cap A_{3^{\prime}}\right)}{\operatorname{det} A_{2^{\prime} 3^{\prime}}}=\frac{Z_{1}\left(Z_{3}+Z_{4}\right)+Z_{3}\left(Z_{2}+Z_{4}\right)}{Z_{1}+Z_{2}+Z_{4}} .
$$

The replacement value $Z_{3}$ of the graph was calculated in accordance with the written conjunction:

$$
Z_{3^{\prime}}=\frac{\operatorname{det}_{Z}\left(A_{1^{\prime}} \cap A_{3^{\prime}}\right)}{\operatorname{det}_{Z} A_{1^{\prime} 3^{\prime}}}=\frac{Z_{1}\left(Z_{2}+Z_{6}\right)+Z_{6}\left(Z_{2}+Z_{4}\right)}{Z_{1}+Z_{2}+Z_{4}} .
$$

The replacement value $Z_{2}$, of the system was calculated in accordance with the written:

$$
Z_{2^{\prime}}=\frac{\operatorname{det}\left(A_{1^{\prime}} \cap A_{2^{\prime}}\right)}{\operatorname{det}_{Z} A_{1^{\prime 2}}}=\frac{Z_{4}\left(Z_{2}+Z_{5}\right)+Z_{5}\left(Z_{1}+Z_{3}\right)}{Z_{1}+Z_{2}+Z_{4}} .
$$

Fig. 4. A substitute system calculated by the structural number method after reduction 
After calculating the substitute values, the correct replacement system was checked (Fig. 4). In the circuit simulation program, the circuits were created, and the branches marked [1]...[6] the impedance values were assigned. From the analysis of presented methods of reduction of the primary system to the replacement system by the method of transfigurations and the method of structural numbers, it follows that the calculated impedance values of individual branches of the replacement system are identical in both methods. This confirms the validity of the presented hypothesis that the presented methods can be used to study and analyze of the piezoelectric systems.

\section{Experimental measurement}

In this point theoretical considerations on the analysis of piezoelectric plates' vibrations will be validated with the conducted experiment. During the generation of characteristics determining the displacement of the piezoelectric stack in the frequency domain, there was used analysis, which main assumption is the distribution of the examined signal into elementary components and to definite the amplitudes of these components.

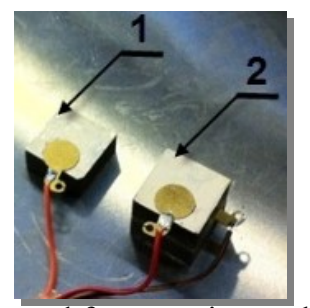

Fig. 5. View of piezoelectric plates used for experimental research

The tests were carried out using the laser vibrometer and the generated signal was recorded using an oscilloscope. The results are shown in the figure 6.

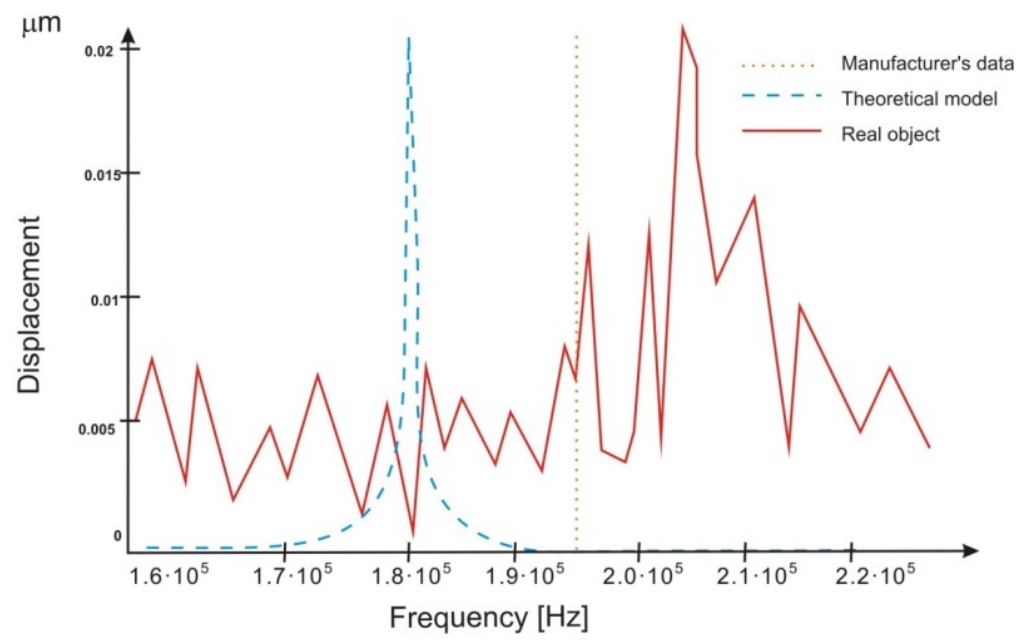

Fig. 6. Comparison of resonant frequencies of the algebraic method and research on the piezoelectric element

The analyzed courses originating from the laser vibrometer showed the vibration courses of the piezoelectric system, whereas for a readable data to be read, there was used a Fast 
Fourier Transform. This analysis allowed to read data in the frequency domain and a readout of the amplitude with given frequency extortion.

\section{Conclusions}

The tests were performed on single piezoelectric elements and the piezoelectric stacks made of two plates. The test results are presented in the form of comparative diagrams. On the basis of the diagrams, there were compared the algebraic method and the research on real elements, but a slight error of both methods was noted. Analyzing the graphs presented in Fig. 6, it was found that the percentage error in relation to the research performed on the real element is not greater than $12 \%$. In the next step, the results of experimental and mathematical models were compared with the data contained in the piezoelectric plates manufacturer's card. The cataloged values of the frequency of vibrations of the tested elements differ about $8 \%$ from the values obtained as a result of testing, and about $7 \%$ in reference to models calculated using the algebraic method. Analyzing the research results, it can be stated that the adopted mathematical model is derived correctly.

\section{References}

1. A. Wróbel A., A. Buchacz, M. Płaczek, Selection of the geometric and materials parameters in piezoelectric sensors level. Vibroengineering PROCEDIA, vol. 10, p. 277-281 (2016)

2. W.P. Mason, Electromechanical Transducers and Wale Filters. Van Nostrand, (1948)

3. A. Buchacz, M. Płaczek, A. Wróbel: Modelling of passive vibration damping using piezoelectric transducers - the mathematical model. Eksploatacja i Niezawodnosc Maintenance and Reliability, 16 (2): 301-306, (2014)

4. S. Bellert, Prace wybrane. Państwowe Wydawnictwo naukowe. Warszawa (1981)

5. W. Soluch (red.), Wstęp do piezoelektroniki. WKi屯, Warszawa 1980,

6. A. Wróbel, M. Płaczek, A. Baier: Calculations of flexibility module in measurements instruments, IOP Conf. Ser.: Mater. Sci. Eng. 227012137 (2017)

7. A. Wróbel, Analysis of possibility of applying the PVDF foil in industrial vibration sensors. Modern technologies in industrial engineering, IOP Conference Series; Materials Science and Engineering ; vol. 95 1757-8981, (2015) 